\title{
FFT of analytically described temperature curves - an investigation of phase angles
}

\author{
By R. Krankenhagen
}

Bundesanstalt für Materialforschung und -prüfung (BAM), 12200 Berlin, Germany, rainer.krankenhagen@bam.de

\begin{abstract}
The Pulsed Phase Thermography (PPT) based on the FFT algorithm is a frequently used method for the evaluation of temperature transients in thermographic investigations, especially for weak and noisy contrasts. However, the applicability of evaluation methods from the field of Lock-in Thermography (LT) is an open issue. Here, this question is considered by means of simple analytical functions for the description of cooling curves. It is shown, that only in case of $2^{n}$ data points the phase values of both PPT as well as LT are equal.
\end{abstract}

\section{Introduction}

Since the introduction of PPT [1], in which the thermal response sequence of a material or layer to a short heating pulse is finally subjected to a Fourier analysis, the observation of phase values has established itself as a common evaluation method. It is particularly useful for very low and noisy thermal contrasts and spatially inhomogeneous temperature distributions. Under certain conditions it is also possible to obtain information about the depth of an inner interface (e.g. contact surface between coating and material or an inner cavity). The quantitative description of the phase difference in relation to a periodic heating pattern is based on the thermal wave model and is the basis of LT. Here, the Bennett-Patty formula [2] can be used to determine the depth of an inner interface if the thermal properties of the inner interface are known. This formula also describes the occurrence of so-called blind frequencies at which the phase contrast between a defective spot and an undamaged area of the same sample completely disappears. Montanini [3] and OswaldTranta [4] each used these formulae for quantitative evaluation of pulse-excited measurements.

However, it is frequently the subject of discussion whether the quantitative evaluation with the Bennett-Patty formula can also be used for phase values that arise as a result of a PPT. Given the linearity of the heat equation, this should be possible without any problems. In 2016 this question was examined in more detail [5] using experimental data of artificial defects in CFRP. Eventually, similarities but also differences were reported.

\section{Analytical approach to describe typical temperature transients}

In order to have a more systematic approach to this question, the difference of 2 simple analytical functions is considered in this paper as a model for the contrast formation after short pulse excitation. $T(t)$ defect describes the temperature curve over a defect and $T(t)_{\text {sound }}$ describes the temperature curve in a region without defect, both in Celsius degree.

$$
\begin{aligned}
& T(t)_{\text {defect }}(t)=e^{-t / 8}+e^{-t / 2.3}+e^{-t / 0.1}+22 \\
& T(t)_{\text {sound }}(t)=e^{-t / 8}+e^{-t / 2.0}+e^{-t / 0.1}+22
\end{aligned}
$$

Fig. 1a shows the corresponding curves and fig. 1b) the difference. It was assumed that a measurement was made with a frame rate of $1 \mathrm{~Hz}$ over a duration of $31 \mathrm{~s}$, starting after the end of a flash excitation with a short pulse duration. Please note the number 32 data points enabling the application of a correct FFT, which requires the number of data point to be $2^{n}$. The aim of this analytical description is not to reproduce a real experiment as accurately as possible. Rather, the aim is to investigate the effect of the tools applied to curves that differ only slightly within a certain time window. 


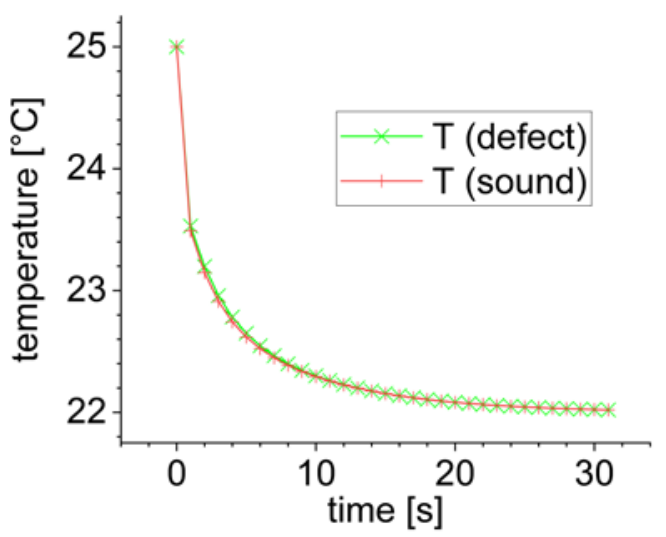

Fig. 1a: signal sequences described by Eq. 1 and Eq. 2 a

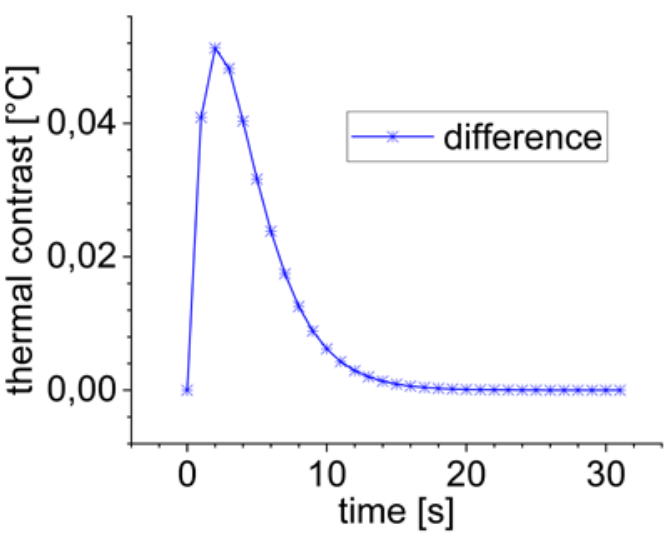

Fig. 1b: difference of both curves in Figure 1 a

\section{Results of FFT analysis and cross correlation analysis}

The Fourier analysis of both "measurement series" was performed by the FFT analysis tool of Origin 2019. The calculated phase difference curve is shown in Fig. 2, it has the usual shape with a pronounced minimum. At higher frequencies the phase differences become small again and then disappear in the noise (indicated here by the hatched area).

The presumed result of a lock-in measurement in the case under consideration is computed by calculating the cross-correlation between signal waveform and a cosine function with the respective lock-in frequency. This approach is based on the consideration that the modelled decay curve in its analytical form represents the system response to a Dirac pulse and thus contains all frequencies. The cross-correlation with the lock-in frequency reaches its maximum when the phase of the lock-in frequency coincides with the frequency-equivalent signal portion of the decay function. The calculation of the maximum of the cross-correlation was realized with Origin 2019 via a non-linear curve fitting. The following function was used as a fit function:

$$
S_{f i t}(t)=S_{0}+A_{0} \cdot \cos (2 \pi f \cdot t-\varphi)
$$

with $S_{0}, A_{0}$, and $\varphi$ as free fit parameters and $f$ is the respective lock-in frequency. Figure 3 shows the fit result for an assumed lock-in frequency of $0.03125 \mathrm{~Hz}$.

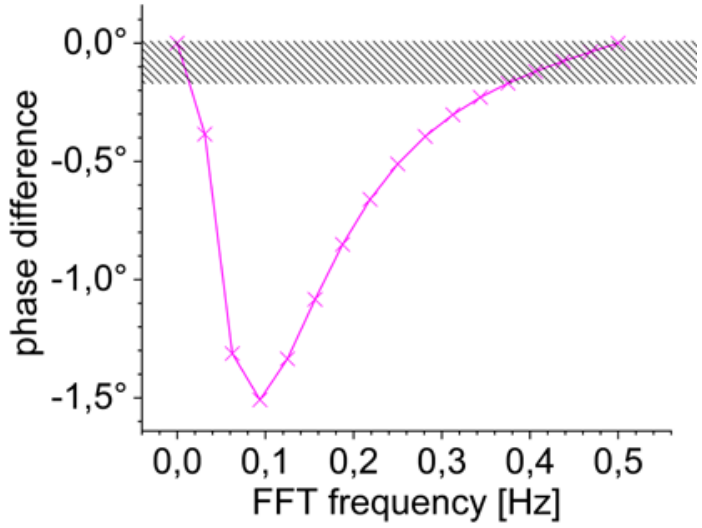

Fig. 2: differences of the phase values calculated by means of FFT applied to $T(t)_{\text {defect }}$ and to $T(t)_{\text {sound }}$

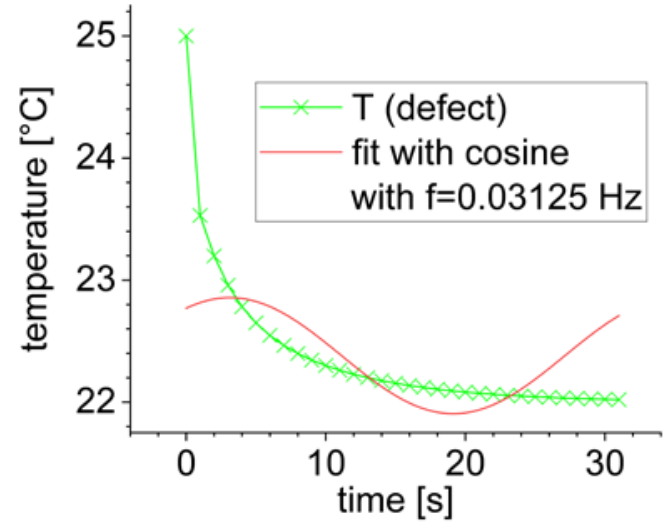

Fig. 3: fitting curve of $T(t)_{\text {defect }}$ using Eq. 3 with fixed frequency, the obtained phase shift $\varphi$ of $35.2^{\circ}$ represents the maximum of the cross correlation between the signal sequence and the cosine function 
The fitting quality is poor as to be expected, because the temperature transient has a completely different shape as the fit function. But this is not the point here. Only the phase shift is of interest, because it represents the expected phase shift in a lock-in measurement with the selected frequency $f$. This analysis was conducted for a series of further lock-in frequencies also present in the FFT spectrum. The direct comparison of these values with those obtained by FFT has practically no deviations in the assumed case $(1 \mathrm{~Hz}$ framerate and 32 data point starting at 0$)$. This means that the applied FFT procedure could determine the correct phase values in this case, which would also be found with a lock-in measurement at the corresponding frequency.

However, a different situation arises if the number of data points is not $2^{n}$. In this case, different phase values are obtained at the respective frequencies. However, the difference between the phase values of $T(t)_{\text {defect }}$ and $T(t)_{\text {sound }}$ corresponds also in this case to the difference resulting from the curve fits (or the respective maxima of the crosscorrelation). This finding will be presented and discussed in the paper.

\section{REFERENCES}

[1] X. Maldague and S. Marinetti, "Pulse phase infrared thermography," Journal of Applied Physics, vol. 79, no. 5 pp. 2694 - 2698, Mar 1 1996, doi: 10.1063/1.362662.

[2] C. A. Bennett and R. R. Patty, "Thermal Wave Interferometry - a Potential Application of the Photo-Acoustic Effect," Appl Optics, vol. 21, no. 1, pp. 49-54, 1982.

[3] R. Montanini, "Quantitative determination of subsurface defects in a reference specimen made of Plexiglas by means of lock-in and pulse phase infrared thermography," Infrared Phys Techn, vol. 53, no. 5, pp. 363-371, Sep 2010, doi: 10.1016/j.infrared.2010.07.002.

[4] B. Oswald-Tranta, "Time and frequency behaviour in TSR and PPT evaluation for flash thermography," Quantitative InfraRed Thermography Journal, pp. 1-21, 2017, doi: 10.1080/17686733.2017.1283743.

[5] C. Maierhofer, M. Rollig, R. Krankenhagen, and P. Myrach, "Comparison of quantitative defect characterization using pulse-phase and lock-in thermography," Appl Optics, vol. 55, no. 34, pp. D76-D86, Dec 1 2016, doi: 10.1364/Ao.55.000d76. 